\section{PSYCAUSE}

Revue scientifique étudiante de

l'École de psychologie de l'Université Laval
UNIVERSITÉ LAVAL

Faculté des sciences sociales École de psychologie

MAI 2019 - VOL. $9 \mathrm{~N}^{\circ} 1$

\title{
EFFETS À LONG TERME DE LA THÉRAPIE COGNITIVE- COMPORTEMENTALE POUR LE TROUBLE DE STRESS POST-TRAUMATIQUE
}

Thalie FLORES ${ }^{1, *}$, Marie-Pier GABOURY ${ }^{1}$, Noémie FISET ${ }^{1}$, Leslie-Ann BOILY ${ }^{1}$ et Geneviève BELLEVILLE

${ }^{1}$ École de psychologie, Université Laval, Québec, Canada

* thalie.flores@hotmail.com

\section{Pour citer l'article}

Flores, T., Gaboury, M.-P., Fiset, N., Boily, L.-A., \& Belleville, G. (2019). Effets à long terme de la thérapie cognitive-comportementale pour le trouble de stress post-traumatique. Psycause: Revue scientifique étudiante de l'École de psychologie de I'Université Laval, 9(1), 14-29.

\section{Droits d'auteur}

(C) 2019 Flores, Gaboury, Fiset, Boily \& Belleville. Cet article est distribué en libre accès selon les termes d'une licence Creative Commons Attribution 4.0 International (de type CC-BY 4.0) qui permet l'utilisation du contenu des articles publiés de façon libre, tant que chaque auteur ou autrice du document original à la publication de l'article soit cité(e) et référencé(e) de façon appropriée. 


\title{
EFFETS À LONG TERME DE LA THÉRAPIE COGNITIVE-COMPORTEMENTALE POUR LE TROUBLE DE STRESS POST-TRAUMATIQUE
}

\author{
Thalie FLORES, Marie-Pier GABOURY, Noémie FISET, Leslie-Ann BOILY et Geneviève BELLEVILLE \\ École de psychologie, Université Laval, Québec, Canada
}

\section{Résumé}

\begin{abstract}
La thérapie cognitive-comportementale (TCC) pour le trouble de stress post-traumatique (TSPT) est validée empiriquement (Forman-Hoffman et al., 2018). Toutefois, à notre connaissance, aucune revue de la littérature ne s'intéresse précisément à l'efficacité à long terme de la TCC du TSPT. Il importe pourtant de s'assurer avec une vision d'ensemble de la durabilité des gains thérapeutiques afin de vérifier si la TCC du TSPT permet d'éviter un retour des symptômes après la thérapie. Des études ont observé que les gains thérapeutiques se maintiendraient entre 6 et 20 mois après la TCC (voir, p. ex., Hembree \& Foa, 2000; Kline, Cooper, Rytwinksi, \& Feeny, 2018) et qu'ils pourraient même s'améliorer (Hembree \& Foa, 2000). La présente revue de littérature identifie des études de traitement, des revues de littérature et des méta-analyses abordant l'efficacité à long terme d'une TCC du TSPT. Ce projet répertorie également les facteurs influençant l'efficacité à long terme d'une TCC individuelle, de groupe et par vidéoconférence. Des articles publiés entre 2010 et 2018 ont été cherchés dans les bases de données MEDLINE et PsycINFO. Deux constats se dégagent de cette revue, soit que la TCC permettrait de traiter le TSPT de façon durable et que certaines variables comme la dépression ou l'anxiété comorbide, un âge avancé, des difficultés de sommeil persistantes et le fait de tarder à aller chercher de l'aide sont associées à une moins bonne efficacité à long terme de la TCC du TSPT. Il est possible que le développement d'habiletés d'adaptation en thérapie soit un facteur de maintien et même d'amélioration des gains après la TCC.
\end{abstract}

Mots-clés : Traitement cognitif-comportemental ou TCC; trouble de stress post-traumatique ou TSPT; efficacité de traitement; maintien des gains thérapeutiques.

\section{Abstract}

Cognitive-behavioural therapy (CBT) for post-traumatic stress disorder (PTSD) is empirically supported (Forman-Hoffman et al., 2018). However, to our knowledge, no literature review specifically addresses the long-term effectiveness of CBT for PTSD. However, it is important to ensure with an overview of the sustainability of therapeutic gains in order to verify whether CBT for PTSD prevents a return of symptoms after therapy. Studies have found that therapeutic gains are maintained between 6 and 20 months after CBT (see, for example, Hembree \& Foa, 2000; Kline, Cooper, Rytwinksi, \& Feeny, 2018) and may even improve (Hembree \& Foa, 2000). This literature review examines treatment studies, literature reviews and metaanalyses that address the long-term effectiveness of CBT for PTSD. This project also identifies factors influencing the long-term effectiveness of individual, group and videoconference-based CBT. Articles published between 2010 and 2018 were searched in the MEDLINE and PsycINFO databases. Two findings emerge from this review, that CBT provides long-term treatment for PTSD and that variables such as comorbid depression or anxiety, advanced age, persistent sleep difficulties and delays in seeking help are associated with a lower long-term effectiveness of CBT for PTSD. It is possible that the development of coping skills in therapy may be a factor in maintaining and even improving gains after CBT.

Mots-clés : Traitement cognitif-comportemental ou TCC; trouble de stress post-traumatique ou TSPT; efficacité de traitement; maintien des gains thérapeutiques.

Au Canada, environ $76 \%$ de la population sera exposée à au moins un événement traumatique (ÉT) au cours de sa vie. Parmi ces personnes, près de $9 \%$ développeront un trouble de stress post-traumatique
(TSPT; Van Ameringen, Mancini, Patterson \& Boyle, 2008). Selon la 5 e édition du Manuel diagnostique et statistique des troubles mentaux (DSM-5; American Psychiatric Association [APA], 2013), le TSPT survient 
en réaction à l'exposition directe ou indirecte à un ou à des ÉT (p. ex., menaces de mort, mort effective, blessures graves, violence sexuelle). Ce diagnostic se caractérise par la présence (1) d'un ou de plusieurs symptômes envahissants, caractérisés par des souvenirs répétitifs et involontaires de l'événement et des rêves répétitifs dont le contenu ou l'affect est lié à l'ÉT; (2) d'un ou de plusieurs symptômes d'évitement des pensées, des souvenirs ou des stimuli liés à l'ÉT; (3) de symptômes d'altération des cognitions et de I'humeur, tels que l'oubli d'un ou de certains aspects importants de l'ÉT et une incapacité à éprouver des émotions positives, et finalement, par la présence (4) de symptômes d'altération de l'éveil et de la réactivité, notamment I'hypervigilance et des réactions de sursaut exagérées (APA, 2013).

Le TSPT est associé à de nombreuses difficultés psychologiques et sociales. Entre autres, des troubles comorbides comme les troubles dépressifs (p. ex., trouble dépressif caractérisé $[O R=5,7]$ ), les troubles anxieux (p. ex., trouble d'anxiété généralisée [OR = $5,3]$ ) et les troubles d'utilisation d'une substance (OR = 2-3,7) sont observés (Possemato, Wade, Andersen \& Ouimette, 2010). Les individus souffrant de TSPT présenteraient aussi un risque six fois plus élevé de faire une tentative de suicide que les personnes présentant un même profil sociodémographique, mais sans ce diagnostic. D'ailleurs, le TSPT est plus associé au suicide que les troubles anxieux (Kessler, 2000; Kessler, Borges \& Walters, 1999). Pacella, Hruska et Delahanty (2013) ont observé une moins bonne santé perçue et plus de problèmes de santé (p. ex., des troubles gastro-intestinaux et des douleurs chroniques) chez les personnes ayant un TSPT. Ce trouble peut aussi engendrer des conséquences sur les relations interpersonnelles comme une augmentation du risque de $60 \%$ de présenter de l'instabilité maritale (Kessler, 2000). De plus, les individus ayant un TSPT peuvent manquer des jours de travail et être moins efficaces dans l'accomplissement de leurs tâches professionnelles comparativement à avant le développement du trouble (Kessler, 2000). Par exemple, dans l'étude de Breslau, Lucia et Davis (2004), les 152 participants ayant un TSPT avaient dû manquer 13,8 jours de travail en moyenne dans les 30 jours où le trouble était en phase aigüe. L'évolution du trouble tend à être chronique; environ $40 \%$ des personnes ayant un TSPT continuent à avoir des symptômes 10 ans après le début du trouble. Cela suggère que les individus ayant un TSPT peuvent subir des conséquences fonctionnelles sur une longue période (Kessler, Sonnega, Bromet, Hughes \& Nelson, 1995). Comme ce trouble entraîne plusieurs conséquences négatives importantes, il faut s'intéresser à traiter efficacement cette problématique et à évaluer si les changements opérés en thérapie peuvent être durables pour l'individu.

La thérapie cognitive-comportementale (TCC) est I'intervention psychologique recommandée en première ligne pour traiter le TSPT (Bisson, Roberts, Andrew, Cooper \& Lewis, 2013; Hembree \& Foa, 2000; International Society for Traumatic Stress Studies, 2015; National Institute for Clinical Excellence (NICE), 2005; U.S. Department of Veterans Affairs, 2017; Watts et coll., 2013). II s'agit d'une approche thérapeutique ciblant la modification des comportements et des pensées problématiques qui maintiennent le trouble. Parmi les TCC utilisées pour le TSPT, il y a l'approche centrée sur le trauma (p. ex., la thérapie cognitive [Cognitive processing therapy] et les traitements basés sur l'exposition), qui est le traitement le plus recommandé pour le TSPT, ainsi que l'approche non centrée sur le trauma (p. ex., interventions de soutien, stratégies basées sur la gestion de l'anxiété et sur la résolution de problèmes, entraînements à la relaxation; Ehring et coll., 2014).

Des composantes de la TCC ont été identifiées comme efficaces pour traiter le TSPT. La restructuration cognitive permet de modifier les croyances erronées entretenant les symptômes de TSPT (Monson \& Schnaider, 2014). Elle cible également les présuppositions et les interprétations erronées des individus qui affectent leurs comportements, leurs attentes, ainsi que l'évaluation qu'ils font des événements (Sherman, 1998). L'exposition permet de tester les distorsions cognitives de la personne par le biais d'expériences comportementales. Elle permet aussi de diminuer l'intensité de la réaction de peur et les stratégies d'évitement (Paintain \& Cassidy, 2018). En effet, l'exposition peut permettre de vivre un processus d'habituation face à l'objet de la peur au fur et à mesure que la personne s'y expose (Monson \& Schnaider, 2014).

La TCC aiderait à diminuer la présence des symptômes de TSPT, d'anxiété et de dépression ainsi qu'à améliorer le fonctionnement (Cusack et coll., 2016). La méta- 
analyse de Bradley, Greene, Russ, Dutra et Westen (2005) montre une diminution des symptômes de TSPT chez la majorité des personnes ayant participé à une TCC impliquant des techniques d'exposition et de restructuration cognitive. D'ailleurs, $62 \%$ des participants ne remplissaient plus les critères diagnostiques du TSPT après avoir suivi une TCC pour ce trouble.

Plusieurs modalités de TCC sont présentement à l'étude pour traiter le TSPT. II est possible d'administrer cette thérapie en face à face ou par vidéoconférence, et elle peut se dérouler de façon individuelle ou en groupe. Plusieurs études soutiennent l'efficacité de ces différentes modalités de la TCC. Par exemple, le traitement de groupe montre une efficacité comparable à la modalité individuelle pour le TSPT (Stalker \& Fry, 1999). Selon la méta-analyse de Knaevelsrud et Maercker (2007), la TCC individuelle administrée par vidéoconférence constituerait une modalité alternative viable à la thérapie en face à face, puisqu'elle permettrait de réduire significativement les symptômes de TSPT. Elle pourrait donc être utile pour pallier les limites contextuelles inhérentes à la psychothérapie en face à face.

Jusqu'à maintenant, aucune revue de la littérature ne s'est précisément penchée sur l'efficacité à long terme des gains thérapeutiques après la TCC du TSPT. Pourtant, vérifier si l'efficacité de cette thérapie est durable pourrait permettre de la rendre plus accessible, ce qui est souhaitable sur le plan de la santé publique, ou encore de proposer des pistes de recherches futures dans le but de l'améliorer. De plus, étant donné que la TCC met l'accent sur le développement d'habiletés d'adaptation que les participants mettent en application par eux-mêmes pour s'améliorer pendant la thérapie (Rector, 2010), il est possible qu'ils continuent de les utiliser après la fin de la thérapie pour maintenir leurs gains thérapeutiques et même continuer à s'améliorer. Ainsi, s'intéresser à l'efficacité à long terme de la TCC du TSPT pourrait permettre de vérifier si cette approche engendre le développement d'habiletés d'adaptation efficaces et durables pour traiter le TSPT.

\section{Objectifs}

La présente revue s'intéresse à dresser un portrait général de la littérature (c.-à-d. études de traitement, méta-analyses, revues de la littérature) sur l'évolution (c.-à-d. maintien, amélioration, détérioration) des gains thérapeutiques à la suite d'une TCC pour le TSPT. Ce projet vise aussi à répertorier les facteurs qui pourraient influencer la présence de symptômes du TSPT après la TCC.

\section{Méthode}

Une revue de la littérature a été complétée dans le but d'explorer l'efficacité à long terme de la TCC du TSPT ainsi que les facteurs qui y sont associés. Une recherche d'articles à l'aide de mots-clés portant sur la TCC du TSPT a été effectuée dans les bases de données PsycINFO et MEDLINE. Les mots-clés utilisés sont: "CBT follow-up», "PTSD», et «Internetbased CBT». Seuls les articles publiés entre 2010 et 2018 ont été sélectionnés par souci de ne présenter que les articles les plus récents.

La sélection de la littérature a été effectuée sur la base de différents critères d'éligibilité. Les critères d'inclusion étaient de (a) présenter les résultats d'une étude de traitement ou uniquement des données de suivi après la TCC du TSPT ou les résultats d'une méta-analyse ou d'une revue de la littérature (c.-à-d. des mesures d'efficacité de la thérapie 1, 3, 6, 12 mois ou toute autre durée après le traitement), et (b) s'intéresser au traitement chez l'adulte (c.-à-d. 18 ans et plus). Toutes les études présentant des résultats d'une TCC individuelle ou de groupe avec la présence physique ou virtuelle d'un thérapeute ont été sélectionnées (Knaevelsrud \& Maercker, 2007; Stalker \& Fry, 1999). Tous les types de protocoles de traitement (c.-à-d. expérimentaux, quasi expérimentaux et études de cas) ont été inclus lorsqu'il s'agissait d'un article présentant les résultats d'une étude de traitement. Le seul critère d'exclusion était de s'intéresser à l'efficacité de la TCC pour le TSPT chez les personnes réfugiées, bien que le TSPT chez ces dernières soit une problématique fortement prévalente-voire au-dessus de la prévalence du TSPT dans la population générale-(Fazel, Wheeler \& Danesh, 2005). Le traitement du TSPT chez les réfugiés est influencé par plusieurs facteurs post-migratoires, tels que le stress 
post-migratoire, la difficulté à trouver un logement ou un emploi satisfaisant, le manque de nourriture, les barrières culturelles et de la langue, la conception de la santé et de la maladie selon la culture (Kirmayer et coll., 2011; Li, Liddell \& Nickerson, 2016). Ainsi, étant donné que plusieurs aspects post-migratoires doivent être considérés dans le traitement de cette population (Kirmayer et coll., 2011), la TCC du TSPT chez les réfugiés diffère de celle administrée aux autres populations touchées par le TSPT. C'est pourquoi nous avons décidé de ne pas inclure les études portant sur les réfugiés dans notre recension.

Le processus de sélection des articles formant l'échantillon de cette revue s'est fait en différentes étapes. Le choix des mots-clés et des bases de données à utiliser a été fait en équipe. Ensuite, à l'aide des mots-clés sélectionnés, la recherche d'articles a été réalisée de façon individuelle dans les bases de données MEDLINE et PsycINFO. Une discussion en équipe a permis de déterminer quels articles allaient être retenus ou non dans l'échantillon. La sélection finale des articles a été effectuée par trois étudiantes au premier cycle et une étudiante au troisième cycle en psychologie. L'étudiante de troisième cycle a finalement gardé une étude dans laquelle les personnes sélectionnées devaient être âgées de 16 ans et plus pour être admises puisque la majorité des participants avaient 18 ans ou plus et l'âge moyen des individus dans l'échantillon était de 22 ans (Hinsberger et coll., 2017).

\section{Résultats}

\section{Caractéristiques des études}

Au total, 27 articles font partie de cette étude. Le Tableau 1 présente le descriptif de ces études. Parmi celles-ci, cinq sont des méta-analyses, 20 sont des études de traitement (dont 10 études randomisées contrôlées, six études d'efficacité de la thérapie en milieu clinique, deux études avec devis quasi expérimentaux, un essai clinique et une étude de traitement avec un seul groupe) et deux sont des revues systématiques de la littérature qui ont été incluses puisqu'elles présentaient des données de suivi après la TCC. Une étude dont les personnes sélectionnées devaient être âgées de 16 ans et plus pour être admises a été incluse puisque l'âge moyen des individus dans l'échantillon était de 22 ans (Hinsberger et coll., 2017). L'ensemble des articles recensés présentent des données quant aux mesures de suivi après une TCC.

Les études de traitement $(n=20)$ varient en ce qui concerne le pays dans lequel a été administré le traitement: le Canada $(n=2)$, la Grande-Bretagne $(n=2)$, I'Espagne $(n=1)$, la Chine $(n=2)$, Israël $(n=1)$, l'Australie $(n=6)$, l'Afrique du Sud $(n=1)$ et les ÉtatsUnis $(n=5)$. Quant aux populations présentant un diagnostic de TSPT, les études recensées indiquent aussi une grande diversité, incluant les vétérans $(n=5)$, les femmes victimes de violence conjugale $(n=1)$, les personnes victimes d'événements traumatiques variés $(n=9)$, le personnel d'urgence $(n=1)$, les victimes d'un accident de la route $(n=1)$, les survivants d'une catastrophe naturelle $(n=1)$, les victimes de violence multiple et raciale $(n=1)$ et, finalement, les victimes d'agression sexuelle $(n=1)$. Il est à noter que $25 \%$ des études incluent dans les critères d'éligibilité des participants qui présentaient soit un ou des troubles anxieux, un trouble du sommeil ou une dépression, tous comorbides au TSPT.

La plupart des études de traitement recensées portaient sur la TCC centrée sur le trauma, dont plusieurs incluent une composante d'exposition au traumatisme $(n=13)$ et deux autres n'incluent que des techniques de restructuration cognitive. Trois études de traitement portaient sur des approches non centrées sur le trauma. Finalement, une étude a comparé une TCC centrée sur le trauma avec une TCC non centrée sur le trauma.

\section{Efficacité à long terme de la TCC du TSPT}

II existe plusieurs études de traitement, des métaanalyses et des revues systématiques de la littérature qui s'intéressent à l'efficacité de la TCC au post-traitement (Barrera, Mott, Hofstein \& Teng, 2013; Murphy \& Smith, 2018). Toutefois, outre la méta-analyse de Kline et coll. (2018), les auteures n'ont pas trouvé de méta-analyse ni de revue systématique abordant spécifiquement l'efficacité à long terme de la TCC du TSPT. Le même constat peut être fait pour ce qui est des études de traitement identifiées. 


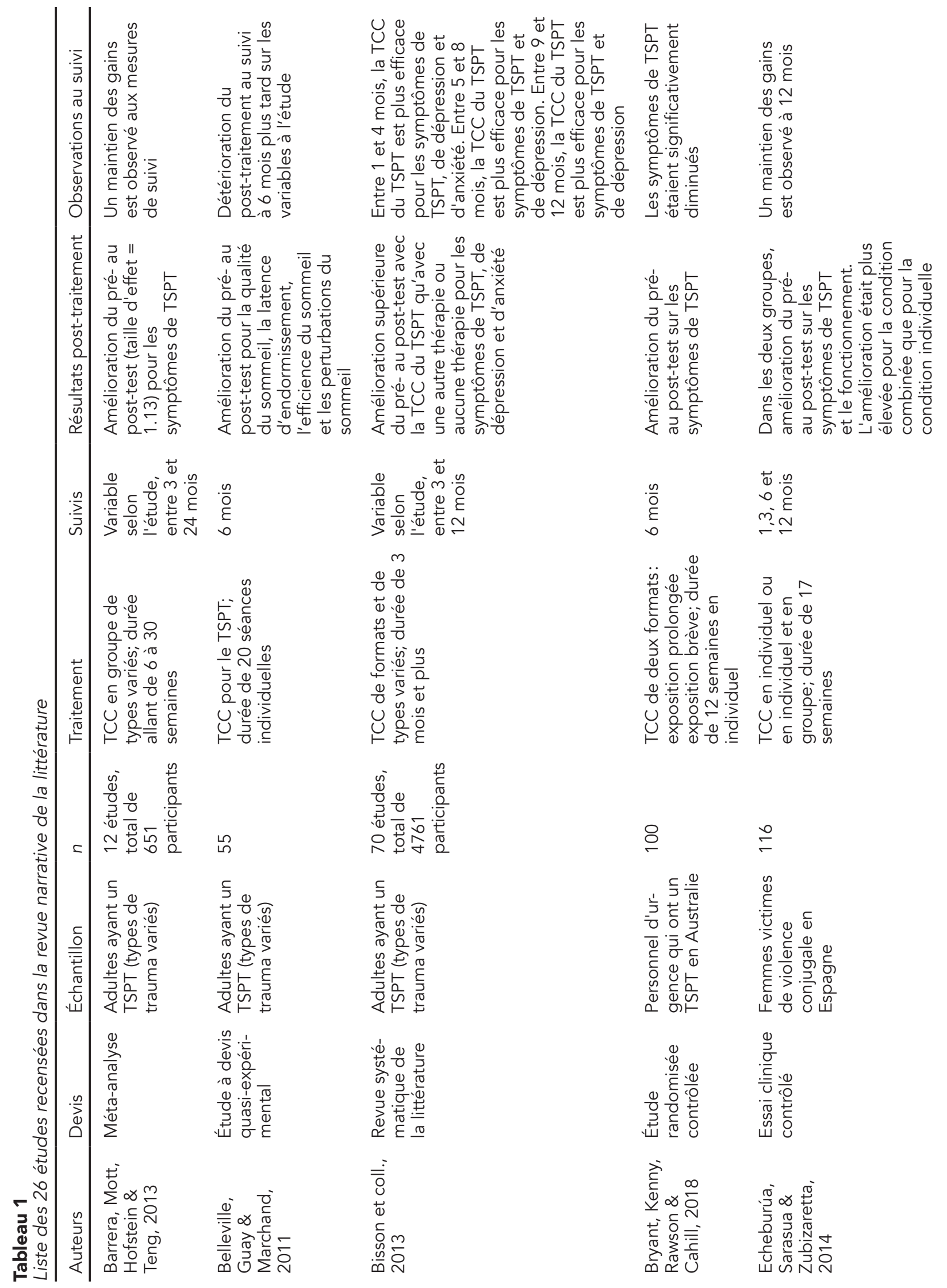




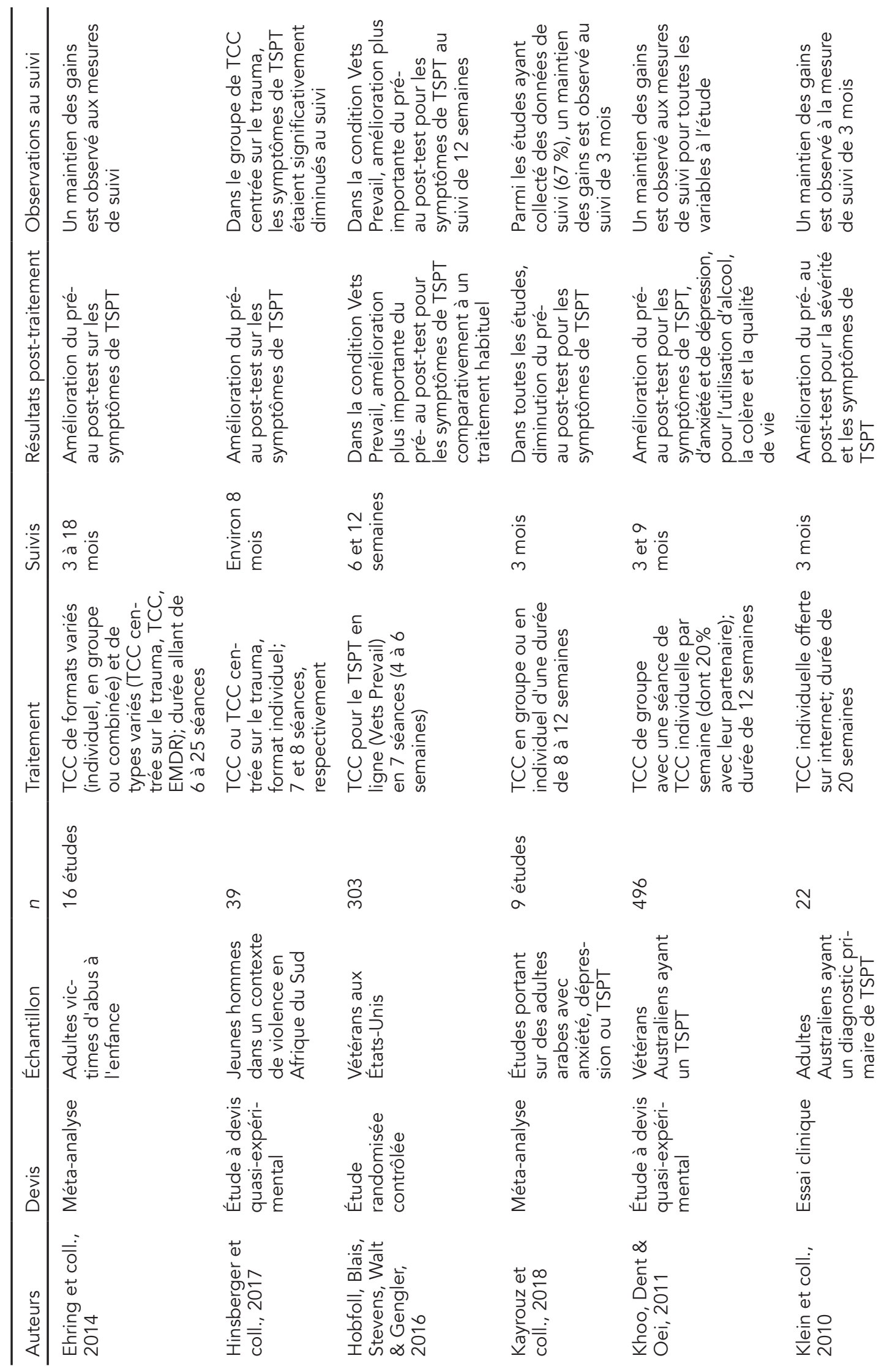




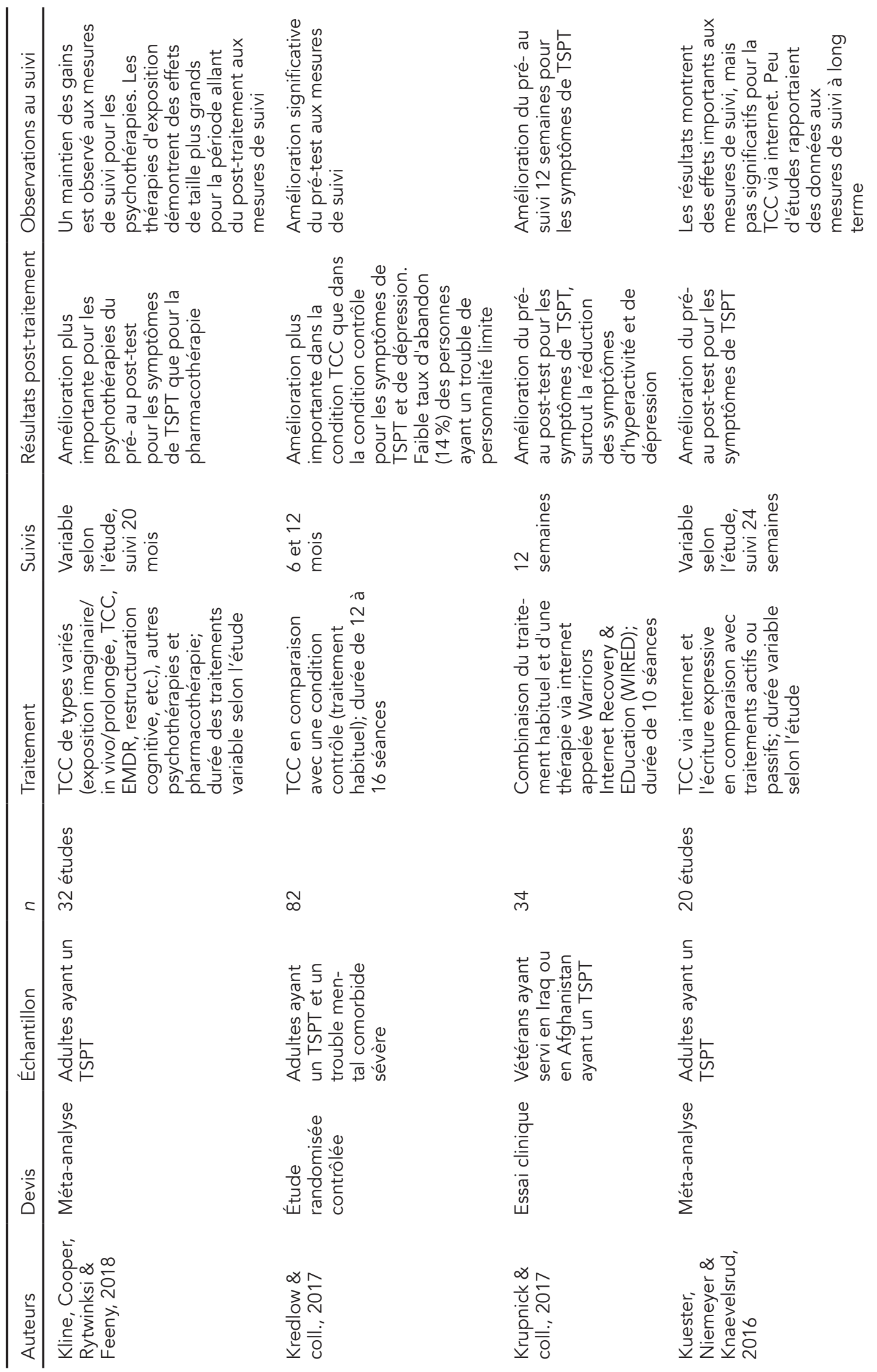




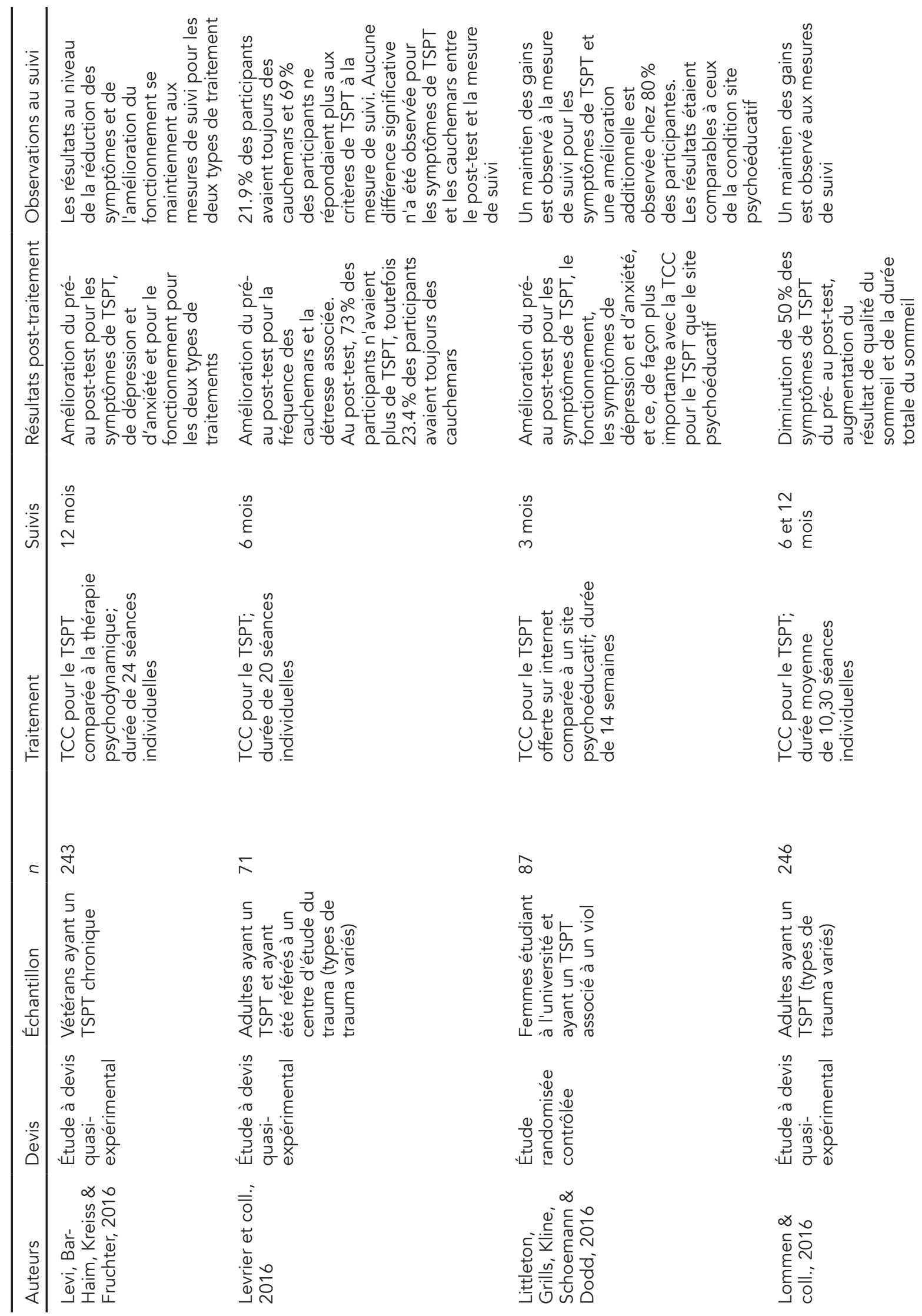




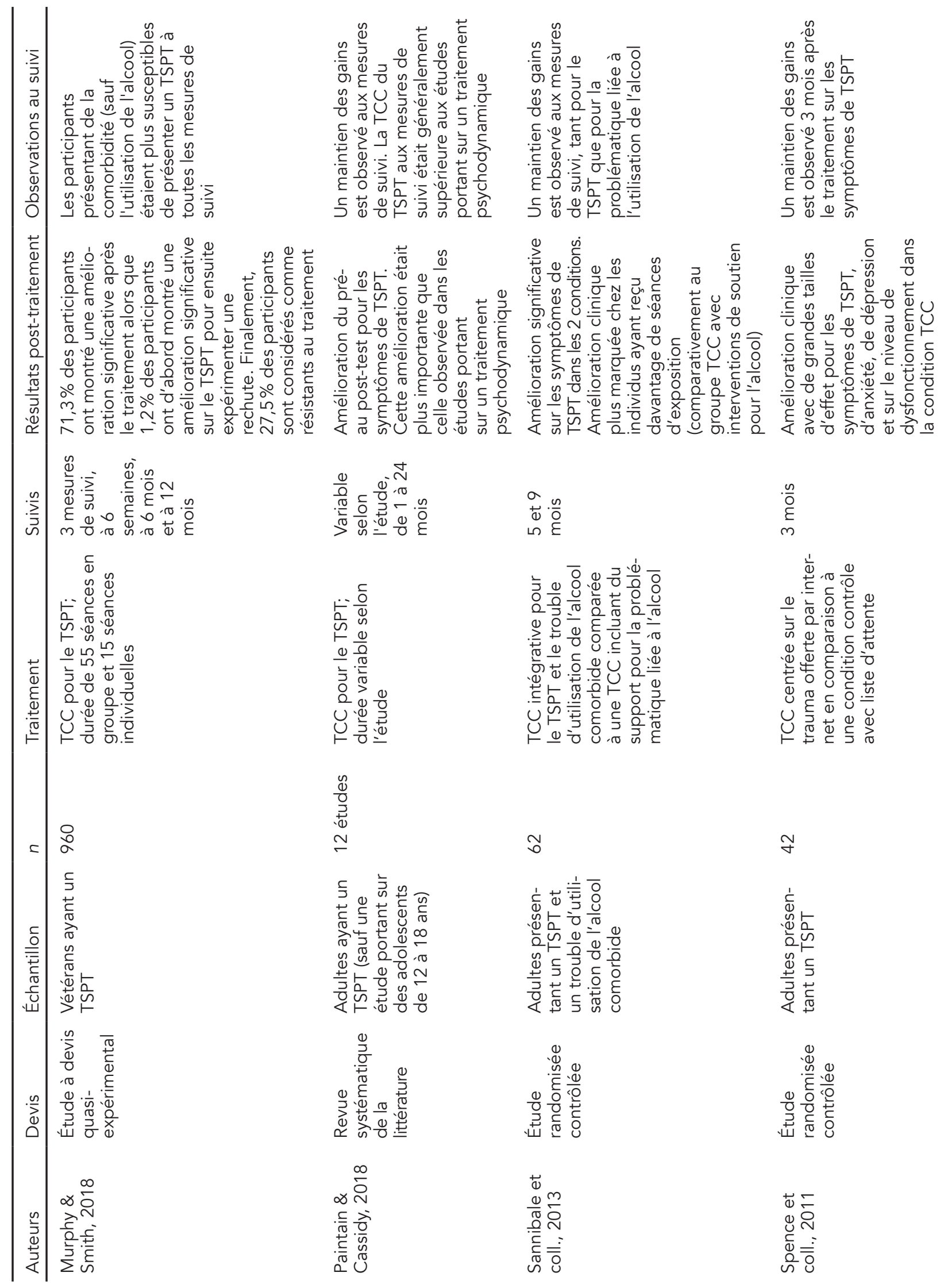




\begin{tabular}{|c|c|c|c|c|}
\hline 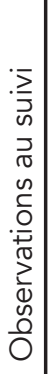 & 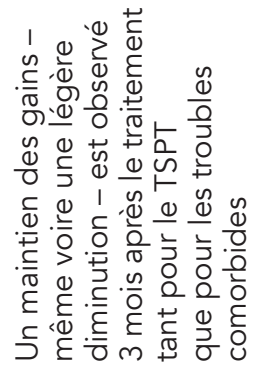 & 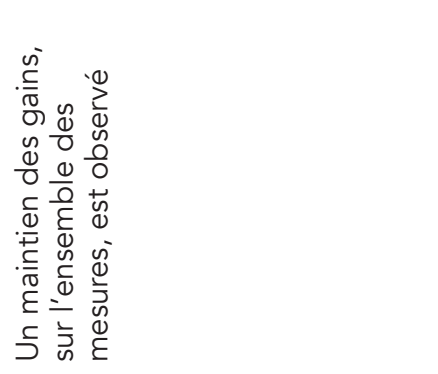 & 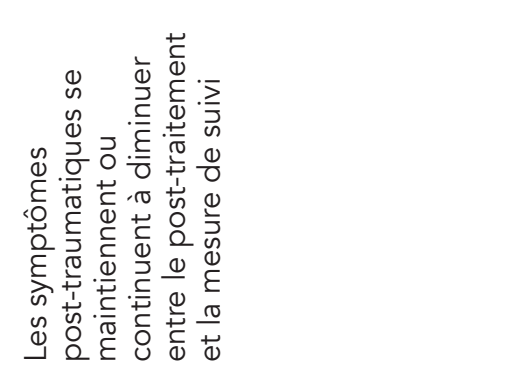 & 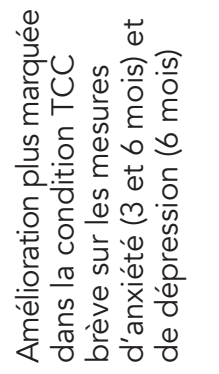 \\
\hline & 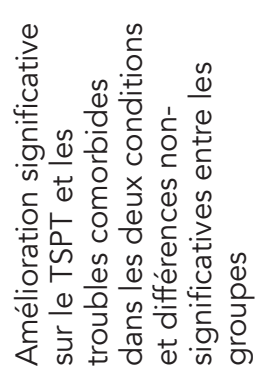 & 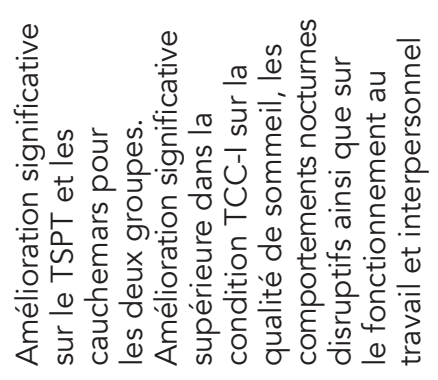 & 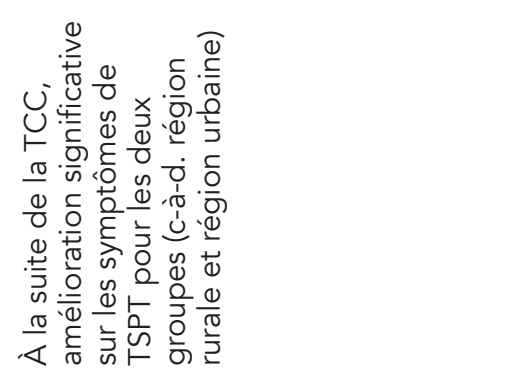 & 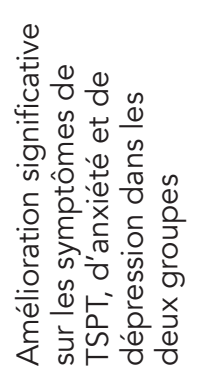 \\
\hline & $\begin{array}{l}\frac{n}{0} \\
\frac{\xi}{m}\end{array}$ & $\begin{array}{l}\frac{n}{0} \\
\frac{\varepsilon}{0}\end{array}$ & $\begin{array}{l}\frac{n}{0} \\
\frac{\varepsilon}{m}\end{array}$ & $\begin{array}{l}0 \\
\stackrel{0}{0} \\
m \\
m\end{array}$ \\
\hline & 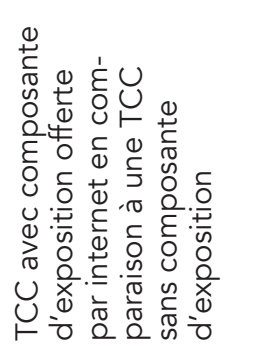 & 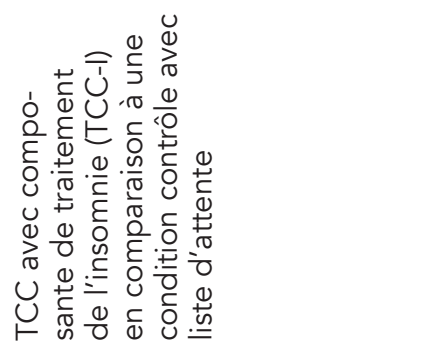 & 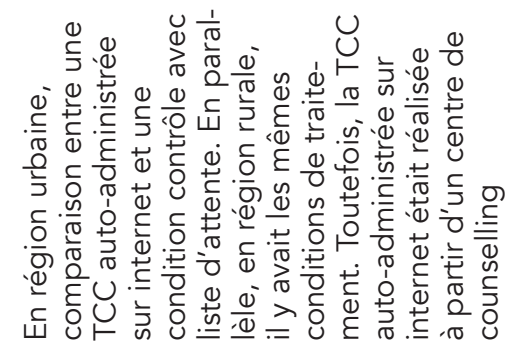 & 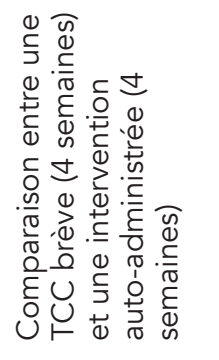 \\
\hline$c$ & $\stackrel{\stackrel{n}{\sim}}{\sim}$ & $\stackrel{\stackrel{\sim}{\forall}}{ }$ & & 이 \\
\hline & 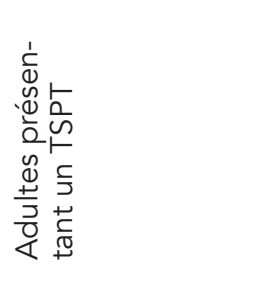 & 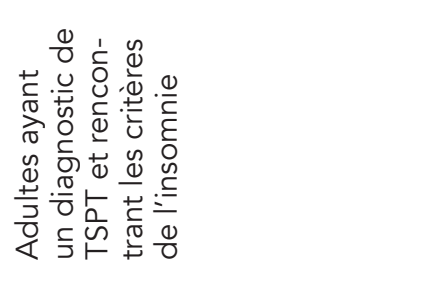 & 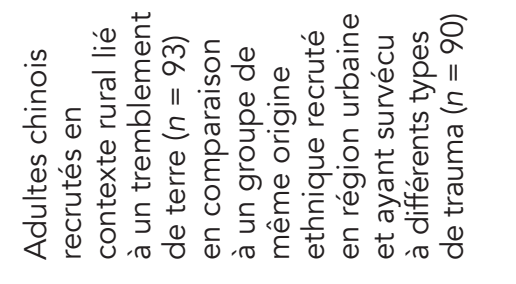 & 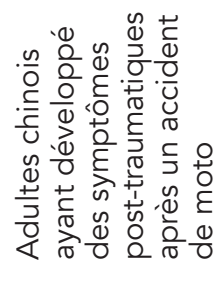 \\
\hline & 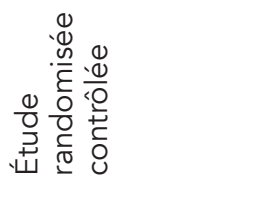 & 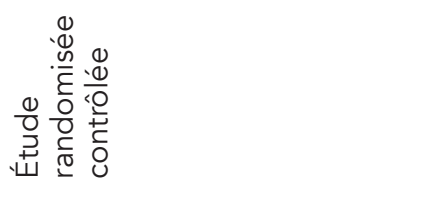 & 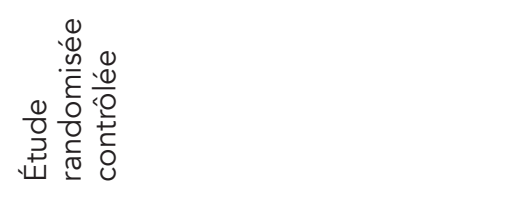 & 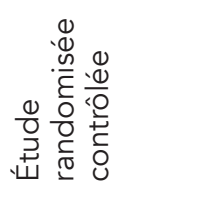 \\
\hline 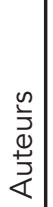 & 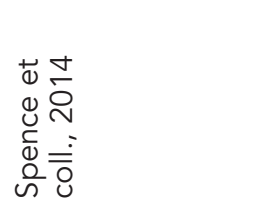 & 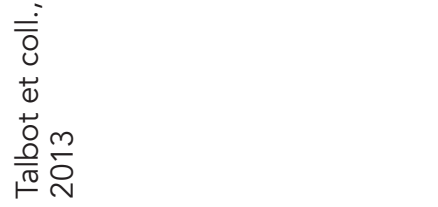 & 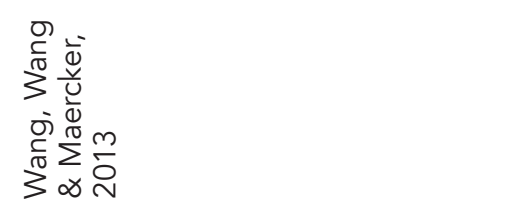 & 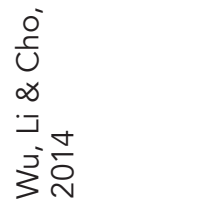 \\
\hline
\end{tabular}


La plupart des études de traitement ainsi que les méta-analyses ont observé que les gains thérapeutiques sur les symptômes de TSPT se maintiennent lors des mesures de suivi. Généralement, les mesures de suivi vont de trois à six mois. La méta-analyse de Bisson et coll. (2013), qui porte sur 70 études, relève que la TCC du TSPT serait efficace à long terme. En effet, les individus ayant bénéficié d'une TCC du TSPT auraient moins de symptômes de TSPT lors des mesures de suivi que des individus ayant bénéficié d'un traitement habituel ou n'ayant bénéficié d'aucun traitement. Une méta-analyse récente portant sur l'efficacité à long terme des psychothérapies pour le TSPT a démontré l'efficacité de la TCC à des temps de suivi à plus long terme, c'est-à-dire allant de six à 20 mois (Kline et coll., 2018). Ces auteurs notent que les traitements centrés sur le trauma et basés sur l'exposition montrent de plus grandes tailles d'effet sur le plan de l'efficacité thérapeutique par rapport aux autres approches de traitement, et ce, entre le post-traitement et les mesures de suivis (c.-à-d. six mois ou plus). Marks, Lovell, Noshirvani, Livanou et Trasher (1998) ont comparé des personnes qui ont reçu différentes approches de TCC du TSPT. Les résultats montrent que les symptômes de TSPT de 47 à $53 \%$ des individus ayant suivi le traitement d'exposition prolongée seule, de restructuration cognitive seule ou ayant suivi les deux traitements combinés ont continué à diminuer jusqu'à six mois après la fin de la thérapie.

\section{Les facteurs liés à l'efficacité à long terme de la TCC du TSPT}

La recension de la littérature révèle que différents facteurs peuvent influencer l'efficacité à long terme de la TCC du TSPT. Murphy et Smith (2018) ont observé que des taux plus élevés de dépression, d'anxiété et de colère chez des vétérans sont associés à une présentation plus importante de symptômes de TSPT 12 mois après le traitement. Pour leur part, Belleville, Guay et Marchand (2011) ont étudié l'effet d'une TCC du TSPT sur le sommeil. Les auteurs ont observé que six mois après une TCC du TSPT, les gains thérapeutiques sur le sommeil, c'est-à-dire sur la qualité du sommeil, sur la latence d'endormissement, sur l'efficacité du sommeil ainsi que sur les difficultés de sommeil (p. ex., des comportements nocturnes perturbateurs et l'insomnie), ne se sont pas maintenus chez tous les participants. II semble que ces difficultés de sommeil persistantes après la fin d'une TCC du TSPT contribuent à une moins bonne efficacité à long terme de la thérapie, puisqu'elles sont associées à des symptômes résiduels de TSPT, d'anxiété et de dépression et à une moins bonne santé mentale et physique. Lommen et coll. (2016) ont toutefois observé que la présence de symptômes de sommeil résiduels à la fin de la TCC du TSPT ne prédisait pas les symptômes de TSPT résiduels à la mesure de suivi (en moyenne, 247 jours après le traitement).

Certaines variables sociodémographiques et temporelles pourraient également influencer le résultat à long terme de la TCC du TSPT. L'étude de Murphy et Smith (2018) a montré qu'un âge plus avancé et que le fait de tarder à aller chercher de l'aide après avoir quitté l'armée sont associés à davantage de symptômes de TSPT qui persistent 12 mois après la fin du traitement.

\section{Discussion}

Les études recensées dans cette revue suggèrent que la TCC permet de traiter le TSPT de façon non seulement efficace, mais également durable. Les études se sont surtout penchées sur la TCC centrée sur le trauma. Il paraît logique que la plupart des études visant à étudier l'efficacité de la TCC s'y soient intéressées, étant donné qu'il s'agit du traitement de première ligne pour le TSPT. Toutes les approches utilisées dans les études de traitement se sont avérées efficaces pour traiter les symptômes de TSPT. En effet, on remarque une diminution de ces symptômes entre le pré- et le post-traitement. De plus, les gains se sont généralement maintenus lors des mesures de suivi. Toutefois, d'autres études montrent une détérioration des symptômes durant les mesures de suivi plutôt qu'un maintien des acquis (Belleville, Dubé-Frenette \& Rousseau, 2018; Murphy \& Smith, 2018). Des facteurs prédisant une moins bonne efficacité à long terme de la TCC du TSPT ont été identifiés, notamment des taux plus élevés de colère, d'anxiété et de dépression, un âge plus avancé, le fait d'avoir tardé à aller chercher de l'aide en quittant l'armée chez le vétéran ainsi que des difficultés de sommeil persistantes (Belleville, Guay \& Marchand, 2011; Murphy \& Smith, 2018). Des variables confondantes pourraient expliquer le fait que ces facteurs sont liés à un moins bon maintien 
des gains thérapeutiques, telles qu'une plus grande sévérité ou chronicité du TSPT ou encore la présence de troubles prémorbides au TSPT (p. ex., insomnie, trouble anxieux ou dépressif). Le fait de vivre des symptômes de TSPT durant une plus longue période pourrait aussi diminuer les ressources disponibles telles que le soutien social et les opportunités d'emploi, ce qui pourrait nuire à l'efficacité à long terme de la TCC du TSPT.

La plupart des études recensées ont observé un maintien des gains lors des mesures de suivi. Ces observations suggèrent que la TCC permet de traiter efficacement et durablement le TSPT. La TCC met l'accent sur le développement d'habiletés d'adaptation, telles que la restructuration cognitive et l'affrontement des problèmes plutôt que l'évitement (Rector, 2010). Les résultats révélant le maintien ou l'amélioration des gains pourraient suggérer que les participants réussissent à continuer à mettre en application ces habiletés après la thérapie.

Cette revue de la littérature comporte des limites dont il faut tenir compte. D'abord, elle n'est pas systématique; elle ne permet pas d'avoir un portrait global des études sur l'efficacité à long terme de la TCC du TSPT. Une sélection limitée de mots-clés et de bases de données a été utilisée pour le choix des articles, ce qui restreint les conclusions pouvant être tirées. De plus, les listes de références des études recensées n'ont pas été consultées, alors que cela aurait pu permettre d'identifier d'autres études pertinentes.

Les études recensées comportent également des limites. Les temps de suivi sont relativement courts; la majorité des mesures varient entre trois et six mois. II n'est donc pas possible, à ce stade de développement de la littérature scientifique, de connaître l'efficacité à plus long terme de la TCC du TSPT. De plus, environ un quart des études ont inclus dans leur échantillon des participants ayant une ou des comorbidités, comme une dépression, un trouble anxieux ou un trouble du sommeil, mais plusieurs études ne traitent pas du facteur de la comorbidité dans leurs résultats. Ainsi, il est possible que la présence de comorbidités influence le maintien des gains dans les mois suivant le traitement. C'est d'ailleurs ce qu'ont observé Murphy et Smith (2018), qui ont relevé qu'une dépression comorbide au TSPT est associée à un moins bon maintien desgains. Toutefois, I'inclusion de participants ayant des comorbidités peut être considérée comme une force des études, étant donné que $83 \%$ des personnes qui ont un TSPT présentent au moins un trouble comorbide (Kessler, 2000). Les échantillons des études ayant inclus la comorbidité sont donc plus représentatifs des clients rencontrés en contexte clinique. Par ailleurs, les populations étudiées dans les études semblent représentatives des populations chez lesquelles le TSPT est plus fréquent, c'est-à-dire les vétérans, les victimes d'agression sexuelle, les victimes de génocides et le personnel d'urgence (APA, 2013), ce qui constitue une force de la présente revue de littérature.

Il faut noter qu'une proportion significative des personnes a complété le traitement et les mesures de suivi dans les études recensées. À titre d'exemple, Bryant et coll. (2018) observent que $86 \%$ des individus ayant terminé le traitement ont complété la mesure de suivi six mois après la thérapie. Malgré cela, I'abandon au cours du traitement et entre la fin du traitement et les mesures de suivi est un phénomène fréquemment observé en contexte clinique chez les personnes qui ont un TSPT. Ce constat se reflète aussi dans les études de traitement recensées (Gutner, Gallagher, Baker, Sloan \& Resick, 2016). II est possible que les participants qui abandonnent diffèrent de ceux qui complètent l'ensemble des mesures de suivi, ce qui peut limiter le caractère généralisable des résultats.

La présente revue de la littérature suggère que les gains acquis au cours de la TCC tendent à se maintenir dans le temps. Toutefois, il semble que certains facteurs associés à l'efficacité à long terme de la TCC du TSPT, tels que des taux plus élevés de colère, l'anxiété et la dépression, un âge plus avancé, le fait d'avoir tardé à aller chercher de l'aide en quittant l'armée chez les vétérans ainsi que des difficultés de sommeil persistantes, demeurent peu identifiés. Comme pistes d'études futures, il serait pertinent, d'une part, de se pencher sur des mesures de suivi à long terme (c.-à-d. de plus de six mois), ce qui permettrait de dresser un portrait plus complet de la durabilité des gains thérapeutiques de la TCC du TSPT. D'autre part, il semble important d'identifier les facteurs associés à l'efficacité à long terme de la TCC du TSPT. Cette identification pourrait permettre d'ajuster certaines interventions cognitives-comportementales pour le TSPT. En effet, les interventions pourraient 
ainsi mieux cibler ces facteurs afin de favoriser un maintien des gains thérapeutiques après la TCC du TSPT. Par exemple, vérifier si le type de trauma visé par la TCC du TSPT influence l'efficacité à long terme du traitement pourrait permettre de bien l'adapter aux clientèles pouvant être touchées par le TSPT.

\section{Références}

American Psychiatric Association. (2013). Diagnostic and Statistical Manual of Mental Disorders - Fifth edition. Washington, DC: Auteur.

Barrera, T. L., Mott, J. M., Hofstein, R. F. \& Teng, E. J. (2013). A meta-analytic review of exposure in group cognitive behavioral therapy for posttraumatic stress disorder. Clinical Psychology Review, 33(1), 24-32. doi:10.1016/j.cpr.2012.09.005.

Belleville, G., Dubé-Frenette, M. \& Rousseau, A. (2018). Efficacy of imagery rehearsal impact therapy and cognitive behavioral therapy in sexual assault victims with posttraumatic stress disorder: A randomized controlled trial. Journal of Traumatic Stress, 31, 591 601. doi:10.1002/jts.22306

Belleville, G., Guay, S. \& Marchand, A. (2011). Persistence of sleep disturbances following cognitive-behavior therapy for posttraumatic stress disorder. Journal of Psychosomatic Research, 70(4), 318-327. doi:10.1016/j.jpsychores.2010.09.022

Bisson, J. I, Roberts, N. P., Andrew, M., Cooper, R. \& Lewis, C. (2013). Psychological therapies for chronic post-traumatic stress disorder (PTSD) in adults. Cochrane Database of Systematic Reviews, 12. doi:10.1002/14651858.CD003388.pub4

Bradley, R., Greene, J., Russ, E., Dura, L. \&Westen, D. (2005). Multi-dimensional meta-analysis of psychotherapy for refugees. American Journal of Psychiatry, 162, 214-227. doi:10.1176/appi.ajp.162.2.214

Breslau, N., Lucia, V. C. \& Davis, G. C. (2004). Partial PTSD versus full PTSD: An empirical examination of associated impairment. Psychological Medicine, 34, 1205-1214. doi:10.1017/S0033291704002594

Bryant, R., Kenny, L., Rawson, N., Cahill, C., Joscelyne, A., Garber, B., ... Nickerson, A. (2018). Efficacy of exposure-based cognitive behaviour therapy for post-traumatic stress disorder in emergency service personnel: A randomised clinical trial. Psychological Medicine, 1-9. doi:10.1017/S0033291718002234
Cusack, K., Jonas, D. E., Forneris, C. A., Wines, C., Sonis, J., Middleton, J. C., ... Gaynes, B. N. (2016). Psychological treatmentsforadultswith posttraumatic stress disorder: A systematic review and metaanalysis. Clinical Psychology Review, 43, 128-141. doi:10.1016/j.cpr.2015.10.003

Echeburúa, E., Sarasua, B. \& Zubizarreta, I. (2014). Individual versus individual and group therapy regarding a cognitive-behavioral treatment for battered women in a community setting. Journal of Interpersonal Violence, 29(10), 1783-1801. doi:10.1177/0886260513511703

Ehring, T., Welboren, R., Morina, N., Wicherts, J. M., Freitag, J. \& Emmelkamp, P. M. (2014). Meta-analysis of psychological treatments for posttraumatic stress disorder in adult survivors of childhood abuse. Clinical Psychology Review, 34(8), 645-657. doi:10.1016/j.cpr.2014.10.004

Fazel, M., Wheeler, J. \& Danesh, J. (2005). Prevalence of serious mental disorder in 7000 refugees resettled in western countries: A systematic review. The Lancet, 365(9467), 1309-1314. doi:10.1016/s0140-6736(05) 61027-6

Forman-Hoffman, V., Middleton, J. C., Feltner, C., Gaynes, B. N., Weber, R. P., Bann, C., ... Green, J. (2018). Psychological and pharmacological treatments for adults with posttraumatic stress disorder: A systematic review update. Agency for Healthcare Research and Quality, Comparative Effectiveness Review, 207. doi:10.23970/AHROEPCCER207

Gutner, C. A., Gallagher, M. W., Baker, A. S., Sloan, D. M. \& Resick, P. A. (2016). Time course of treatment dropout in cognitive-behavioral therapies for posttraumatic stress disorder. Psychological Trauma: Theory, Research, Practice, and Policy, 8(1), 115121. doi: $10.1037 /$ tra0000062

Hembree, E. A. \& Foa, E. B. (2000). Posttraumatic stress disorder: Psychological factors and psychosocial interventions. The Journal of Clinical Psychiatry, 61(7), 33-39.

Hinsberger, M., Holtzhausen, L., Sommer, J., Kaminer, D., Elbert, T.,Seedat, S., ... Weierstall, R. (2017). Feasibility and effectiveness of narrative exposure therapy and cognitive behavioral therapy in a context of ongoing violence in South Africa. Psychological Trauma: Theory, Research, Practice, and Policy, 9(3), 282291. doi:10.1037/tra0000197 
Hobfoll, S. E., Blais, R. K., Stevens, N. R., Walt, L. \& Gengler, R. (2016). Vets prevail online intervention reduces PTSD and depression in veterans with mild-to-moderate symptoms. Journal of Consulting and Clinical Psychology, 84(1), 31-42. doi:10.1037/ cсp0000041

International Society for Traumatic Stress Studies. (2015). Posttraumatic stress disorder prevention and treatment guideline - Methodology and recommendations. Oakbrook Terrace, IL: International Society for Traumatic Stress Studies. Récupéré de http://www.istss.org/ getattachment/Treating-Trauma/New-ISTSS-Prevention-and-Treatment-Guidelines/ISTSS_PreventionTreatmentGuidelines_FNL.pdf.aspx

Kayrouz, R., Dear, B. F., Kayrouz, B., Karin, E., Gandy, M. \& Titov, N. (2018). Meta-analysis of the efficacy and acceptability of cognitive-behavioural therapy for Arab adult populations experiencing anxiety, depression or post-traumatic stress disorder. Cognitive Behaviour Therapy, 47(5), 412-430. doi:10. 1080/16506073.2018.1445124

Kessler, R. C. (2000). Posttraumatic stress disorder: The burden to the individual and to society. Journal of Clinical Psychiatry, 61(5), 4-12.

Kessler, R. C., Borges, B. \&Walters, E. E. (1999). Prevalence of and risk factors for lifetime suicide attempts in the National Comorbidity Survey. Archives of General Psychiatry, 56(7), 617-626. doi:10.1001/ archpsyc.56.7.617

Kessler, R. C., Sonnega, A., Bromet, E., Hughes, M. \& Nelson, C. B. (1995). Posttraumatic stress disorder in the National Comorbidity Survey. Archives of General Psychiatry, 52(12), 1048-1060. doi:10.1001/ archpsyc.1995.03950240066012

Khoo, A., Dent, M. T. \& Oei, T. P. (2011). Group cognitive behaviour therapy for military service-related posttraumatic stress disorder: Effectiveness, sustainability and repeatability. Australian and New Zealand Journal of Psychiatry, 45(8), 663-672. doi:10.3109/00048 674.2011.590464

Kirmayer, L. J., Narasiah, L., Munoz, M., Rashid, M., Ryder, A.G., Guzder, J., ... Pottie, K. (2011). Common mental health problems in immigrants and refugees: General approach in primary care. Canadian Medical Association Journal, 183(12), 959-967. doi:10.1503/ cmaj.090292
Klein, B., Mitchell, J., Abbott, J., Shandley, K., Austin, D., Gilson, K., ... Redman, T. (2010). A therapistassisted cognitive behavior therapy Internet intervention for posttraumatic stress disorder: Pre-, post- and 3-month follow-up results from an open trial. Journal of Anxiety Disorders, 24(6), 635-644. doi:10.1016/j. janxdis.2010.04.005

Kline, A. C., Cooper, A. A., Rytwinksi, N. K. \& Feeny, N. C. (2018). Long-term efficacy of psychotherapy for posttraumatic stress disorder: A meta-analysis of randomized controlled trials. Clinical Psychology Review, 59, 30-40. doi:10.1016/j.cpr.2017.10.009

Knaevelsrud, C. \& Maercker, A. (2007). Internet-based treatment for PTSD reduces distress and facilitates the development of a strong therapeutic alliance: $A$ randomized controlled clinical trial. BMC Psychiatry, 7(13). doi:10.1186/1471-244X-7-13

Kredlow, M. A., Szuhany, K. L., Lo, S., Xie, H., Gottlieb, J. D., Rosenberg, S. D. \& Mueser, K. T. (2017). Cognitive behavioral therapy for posttraumatic stress disorder in individuals with severe mental illness and borderline personality disorder. Psychiatry Research, 249, 86-93. doi:10.1016/j.psychres.2016.12.045

Krupnick, J. L., Green, B. L., Amdur, R., Alaoui, A., Belouali, A., Roberge, E., ... Dutton, M. A. (2017). An Internet-based writing intervention for PTSD in veterans: A feasibility and pilot effectiveness trial. Psychological Trauma: Theory, Research, Practice, and Policy, 9(4), 461-470. doi:10.1037/tra0000176

Kuester,A., Niemeyer,H.\&Knaevelsrud,C.(2016). Internetbased interventions for posttraumatic stress: A meta-analysis of randomized controlled trials. Clinical Psychology Review, 43, 1-16. doi:10.1016/j. cpr.2015.11.004

Levi, O., Bar-Haim, Y., Kreiss, Y. \& Fruchter, E. (2016). Cognitive-behavioural therapy and psychodynamic psychotherapy in the treatment of combat-related post-traumatic stress disorder: A comparative effectiveness study. Clinical Psychology \& Psychotherapy, 23(4), 298-307. doi:10.1002/cpp.1969

Levrier, K., Marchand, A., Belleville, G., Dominic, B. P. \& Guay, S. (2016). Nightmare frequency, nightmare distress and the efficiency of trauma-focused cognitive behavioral therapy for post-traumatic stress disorder. Archives of Trauma Research, 5(3), 1-6. doi:10.5812/ atr.33051 
Li, S.S., Liddell, B.J.\&Nickerson, A. (2016). The relationship between post-migration stress and psychological disorders in refugees and asylum seekers. Current Psychiatry Reports, 18(82). doi:10.1007/s11920016-0723-0

Littleton, H., Grills, A. E., Kline, K. D., Schoemann, A. M. \& Dodd, J. C. (2016). The From Survivor to Thriver Program: RCT of an online therapist-facilitated program for rape-related PTSD. Journal of Anxiety Disorders, 43, 41-51. doi:10.1016/j.janxdis.2016.07.010

Lommen, M. J., Grey, N., Clark, D. M., Wild, J., Stott, R. \& Ehlers, A. (2016). Sleep and treatment outcome in posttraumatic stress disorder: Results from an effectiveness study. Depression and Anxiety, 33, 575-583. doi:10.1002/da.22420

Marks, I., Lovell, K., Noshirvani, H., Livanou, M. \& Trasher, S. (1998). Treatment of posttraumatic stress disorder by exposure and/or cognitive restructuring. Archives of General Psychiatry, 55(4), 317-325. doi:10.1001/archpsyc.55.4.317

Monson, C. M. \& Shnaider, P. (2014). Treating PTSD with cognitive-behavioral therapies: Interventions that work. Washington, DC: American Psychological Association. doi:10.1037/14372-000

Murphy, D. \& Smith, K. V. (2018). Treatment efficacy for veterans with posttraumatic stress disorder: Latent class trajectories of treatment response and their predictors. Journal of Traumatic Stress, 31(5), 753763. doi:10.1002/jts.22333

National Institute for Clinical Excellence (2005). Posttraumatic stress disorder: Management (CG26). Manchester, UK: National Institute for Health and Clinical Excellence. Récupéré de www.nice.org.uk/ guidance.cg26

Pacella, M. L., Hruska, B. \& Delahanty, D. L. (2013). The physical health consequences of PTSD and PTSD symptoms: A meta-analytic review. Journal of Anxiety Disorders, 27(1), 33-46. doi:10.1016/j.janxdis.2012.08.004

Paintain, E. \& Cassidy, S. (2018). First-line therapy for post-traumatic stress disorder: A systematic review of cognitive behavioural therapy and psychodynamic approaches. Counselling and Psychotherapy Research, 18(3), 237-250. doi:10.1002/capr.12174
Possemato, K., Wade, M., Andersen, J. \& Ouimette, P. (2010). The impact of PTSD, depression, and substance use disorders on disease burden and health care utilization among OEF/OIF veterans. Psychological Trauma: Theory, Research, Practice, and Policy, 2, 218-223. doi:10.1037/a0019236

Rector, N. A. (2010). La thérapie cognitivo-comportementale: guide d'information. Toronto, Canada: Centre de toxicomanie et de santé mentale.

Sannibale, C., Teesson, M., Creamer, M., Sitharthan, T., Bryant, R. A., Sutherland, K., ... Peek-O'Leary, M. (2013). Randomized controlled trial of cognitive behaviour therapy for comorbid post-traumatic stress disorder and alcohol use disorders. Addiction, 108(8), 1397-1410. doi:10.1111/add.12167

Sherman, J. J. (1998). Effects of psychotherapeutic treatments for PTSD: A meta-analysis of controlled clinical trials. Journal of Traumatic Stress, 11(3), 413435. doi:10.1023/A:1024444410595

Spence, J., Titov, N., Dear, B. F., Johnston, L., Solley, K., Lorian, C., ... Schwenke, G. (2011). Randomized controlled trial of Internet-delivered cognitive behavioral therapy for posttraumatic stress disorder. Depression and Anxiety, 28(7), 541-550. doi:10.1002/da.20835

Spence, J., Titov, N., Johnston, L., Jones, M. P., Dear, B. F. \& Solley, K. (2014). Internet-based traumafocused cognitive behavioural therapy for PTSD with and without exposure components: A randomised controlled trial. Journal of Affective Disorders, 162, 73-80. doi:10.1016/j.jad.2014.03.009

Stalker, C. A. \& Fry, R. (1999). A comparison of short-term group and individual therapy for sexually abused women. The Canadian Journal of Psychiatry, 44(2), 168-174. doi:10.1177/070674379904400208

Talbot, L. S., Maguen, S., Metzler, T. J., Schmitz, M., McCaslin, S. E., Richards, A., ... Neylan, T. C. (2014). Cognitive behavioral therapy for insomnia in posttraumatic stress disorder: A randomized controlled trial. Sleep, 37(2), 327-341. doi:10.5665/sleep.3408

U.S. Department of Veterans Affairs. (2017). Management of posttraumatic stress and acute stress reactions. Récupéré de http://www.healthquality.va.gov/Post_ Traumatic_Stress_Disorder_PTSD.asp

Van Ameringen, M., Mancini, C., Patterson, B. \& Boyle, M. H. (2008). Post-traumatic stress disorder in Canada. CNS Neuroscience \& Therapeutics, 14, 71-181. doi:10.1111/j.1755-5949.2008.00049.x 
Wang, Z., Wang, J. \& Maercker, A. (2013). Chinese My Trauma Recovery, a Web-based intervention for traumatized persons in two parallel samples: Randomized controlled trial. Journal of Medical Internet Research, 15(9). doi:10.2196/jmir.2690

Watts, B., Shnurr, P., Mayo, L., Young, Y., Weeks, W. \& Friedman, M. (2013). Meta-analysis of the efficacy of treatments for posttraumatic stress disorder. Journal of Clinical Psychiatry, 74(6), 541-550. doi:10.4088/ JCP.12r08225
Wu, K. K., Li, F. W. \& Cho, V. W. (2014). A randomized controlled trial of the effectiveness of brief-CBT for patients with symptoms of posttraumatic stress following a motor vehicle crash. Behavioural and Cognitive Psychotherapy, 42(1), 31-47. doi:10.1017/ S1352465812000859

\section{Pour citer l'article}

Flores, T., Gaboury, M.-P., Fiset, N., Boily, L.-A., \& Belleville, G. (2019). Effets à long terme de la thérapie cognitivecomportementale pour le trouble de stress post-traumatique. Psycause : Revue scientifique étudiante de l'École de psychologie de l'Université Laval, 9(1), 14-29.

\section{Droits d'auteur}

(C) 2019 Flores, Gaboury, Fiset, Boily \& Belleville. Cet article est distribué en libre accès selon les termes d'une licence Creative Commons Attribution 4.0 International (de type CC-BY 4.0) qui permet l'utilisation du contenu des articles publiés de façon libre, tant que chaque auteur ou autrice du document original à la publication de l'article soit cité(e) et référencé(e) de façon appropriée. 